\title{
Arbeitsforschung 2021+: Welche Forschungsfragen bewegen die Arbeitgeber und wie sieht die Arbeitswelt der Zukunft aus?
}

\author{
Bundesvereinigung der Deutschen Arbeitgeberverbände (BDA) ${ }^{1}$ \\ Angenommen: 10. Februar 2021 / Online publiziert: 5. März 2021 \\ (c) Springer-Verlag GmbH Deutschland, ein Teil von Springer Nature 2021
}

\section{Zusammenfassung}

Der Arbeitskreis „Arbeitsgestaltung und -forschung“ der Bundesvereinigung der Deutschen Arbeitgeberverbände (BDA) beschreibt aus einer praxisnahen Perspektive verschiedener deutscher Industrie- und Dienstleistungsbranchen heraus wichtige Forschungsbedarfe für die Arbeitswelt der kommenden Jahre (2021+). Ziel ist es, die vielfältigen Facetten und den Wandel der Arbeitswelt zu beschreiben, relevante Positionen und Thesen für die Arbeitsforschung aus Arbeitgebersicht herzuleiten sowie die Diskussion zur Weiterentwicklung der Arbeitswissenschaft anzuregen. Betrachtet werden soziotechnische Aspekte, wie zum Beispiel die Auswirkung der Digitalisierung und Künstlichen Intelligenz auf die Arbeitsgestaltung, Nachhaltigkeit in der Wirtschaft. Verschiedene arbeitsorganisatorische Ausprägungen, wie zum Beispiel mobile Arbeit, agiles Arbeiten, Führungsanforderungen, stehen ebenso im Mittelpunkt der Thesen.

Schlüsselwörter Arbeitsforschung · Arbeitswelt der Zukunft · Digitalisierung · Arbeitgeberverbände · Wandel

\section{Work research 2021+: What research questions are employers moving and what will the future of work look like?}

\begin{abstract}
The working group "Work Design and Research" of the Confederation of German Employers' Associations (BDA) describes important research needs for a working environment in the coming years $(2021+)$ from a practical perspective of various German industrial and service sectors. The aim is to describe the many facets and changes in the world of work, to derive relevant positions and theses for work research from the employer's point of view, and to stimulate discussion on the further development of work science. Socio-technical aspects are considered, such as the impact of digitization and artificial intelligence on work design, sustainability in the economy. Different work organization characteristics, such as mobile work, agile work, leadership requirements, are also the focus of the theses.
\end{abstract}

Keywords Work research $\cdot$ The world of work of the future $\cdot$ Digitization $\cdot$ Employers' associations $\cdot$ Change

Die BDA organisiert als Spitzenverband die sozial- und wirtschaftspolitischen Interessen der gesamten deutschen Wirtschaft. Wir bündeln die Interessen von einer Million Betrieben mit rund 20 Mio. Beschäftigten. Diese Betriebe sind der BDA durch freiwillige Mitgliedschaft in Arbeitgeberverbänden verbunden.

Bundesvereinigung der Deutschen Arbeitgeberverbände

(BDA)

soziale.sicherung@arbeitgeber.de

1 Breite Straße 29, 10178 Berlin, Deutschland

\section{Vom Wandel der Arbeitswelt und den Fragen, die sich daraus ergeben}

Innerhalb der letzten 50 Jahre ist unsere Lebens- und Arbeitswelt um ein Vielfaches flexibler und digitaler geworden, Innovationszyklen werden immer kürzer. Faktoren wie die Digitalisierung, der demografische Wandel, die Globalisierung und ein Wertewandel in der Gesellschaft bewirken eine stetige Anpassung und Veränderung. Unternehmen müssen sich in Flexibilität üben und sich kontinuierlich verändern, um ihre Existenz zu sichern. Ein Teil der Unternehmen ist zu einem ,permanenten Wandel“ übergegangen - 
und es stellt sich die Frage, wie dieser gut gestaltet werden kann. Im Rahmen der stetigen Veränderungen gewinnen Arbeitsformen wie agiles, orts- bzw. zeitflexibles und digitales (und die Kombination aus diesen) Arbeiten an immer mehr Bedeutung. Diese Formen des Arbeitens fordern neue Arten der Führung und der Selbstorganisation. Führung muss heute ebenfalls aus der Distanz effektiv erfolgen.

Im Arbeitskontext ist hierbei die Digitalisierung längst angekommen: Videokonferenzen, virtuelle Abstimmungstools, Arbeiten in der Cloud und mit Algorithmen bzw. Chatbots sowie mit den modernsten Produktionsmaschinen sind nichts Außergewöhnliches mehr. Eine weitere technische Entwicklungsstufe ist die Künstliche Intelligenz. Sie ermöglicht es den Beschäftigten mit intelligenter Assistenz, lernenden Robotern und benutzeroptimierter Informationsbereitstellung noch flexibler zu arbeiten, anspruchsvollere Tätigkeiten zu übernehmen, individuell angepasste Informationen zu erhalten sowie bei monotonen geistigen Routinetätigkeiten entlastet zu werden. Neben technischen Innovationen werden zunehmend Faktoren wie Nachhaltigkeit, Wertewandel und neue Arbeitsformen die Arbeitswelt der Zukunft bestimmen. Die Bedeutung von Eigenverantwortung und lebensbegleitendes Lernen nimmt weiter zu und ist nicht nur Herausforderung für Beschäftigte, sondern auch für Unternehmen, die ihre Beschäftigten bei diesen Themen unterstützen wollen. $\mathrm{Zu}$ diesen langfristigen Trends kommt die Erschütterung durch die SARS-CoV-2-Pandemie hinzu. Diese Krise konnte aufzeigen, wo Unternehmen Flexibilisierungs- und Digitalisierungspotenzial aufweisen, und an welchen Stellen Unternehmen bereits gut aufgestellt sind.

Dieses Papier verdeutlicht aus einer Praxisperspektive Forschungsbedarfe für eine Arbeitswelt in den kommenden Jahren (2021+). Aus der Bestandsaufnahme ergeben sich zudem zentrale Thesen, die sich zusammengefasst am Ende des Papiers befinden. Ziel ist es, die Arbeit der $\mathrm{Zu}-$ kunft gut und sicher zu gestalten und dabei für die Unternehmen und Beschäftigten die passenden Rahmenbedingungen zu schaffen. Damit dies gelingt, müssen aus Sicht der Unternehmen noch einige zentrale Fragen beantwortet werden und auch die Bedarfe von Kleinst- und Kleinunternehmen (KKU) sowie mittelständischen Betrieben Berücksichtigung finden. Insbesondere bei Letzteren muss in den Forschungsprojekten sichergestellt werden, dass die Ergebnisse auf KKU übertragbar sind.

\section{Permanenter Wandel wird Alltag}

Veränderungsmanagement muss sich selbst einem Wandel unterziehen (Albach et al. 2014). Veränderungen laufen anders ab als noch vor 10 oder 20 Jahren und viele Empfehlungen für gute Veränderungsprozesse wären heute kontraproduktiv. Damals ging man noch von einzelnen, klar definierten Veränderungen aus, zwischen denen sich eine neue Routine herausbilden kann, und Beschäftigte sich auf die neue Situation einstellen können, weil sich ein neues Gleichgewicht herausbildet. Diese negative Sicht auf Veränderungen als Belastung der Beschäftigten und positive Bewertung von Phasen ohne Veränderungen als gute und präventive Arbeitsgestaltung lässt sich so nicht durchhalten.

Externe Faktoren wie technologische Entwicklungen und Trends sowie interne Faktoren wie wechselndes Management mit wechselnden Strategien und Führungskulturen fordern Unternehmen ständige Anpassungen und vorausschauende Veränderungen $a b$, wenn sie überleben wollen. Mit permanentem Wandel sind in diesem Papier eher weniger die seit Anfang der 1990er-Jahre gut etablierten kontinuierlichen Verbesserungsprozesse (KVP) gemeint, die mit strukturiertem Vorgehen Verbesserungen im Unternehmen vorantreiben. Vielmehr geht es um Wandel, der mit wenig Vorlauf und Absehbarkeit einhergeht. Einige Erkenntnisse des „klassischen“ Veränderungsmanagements sind für einige Unternehmen nicht mehr von Relevanz, die durch viele weniger klar als Projekt abgrenzbare Veränderungen quasi in einen permanenten Wandel übergegangen sind.

Die Gestaltung von Wandel ist zunehmend zur Daueraufgabe geworden, und eine strikte Einteilung in Phasen klar abgegrenzter Veränderungen mit Handlungsempfehlungen für die einzelnen Phasen ist nur noch teilweise hilfreich. Für die zunehmende Zahl an Unternehmen für die Wandel zur Daueraufgabe geworden ist, müssen Erkenntnisse generiert und Handlungsempfehlungen abgeleitet werden, wie solch ein permanenter Wandel gut und gesundheitsgerecht gestaltet werden kann. Eine ablehnende Haltung zu Veränderungen wäre genauso wenig hilfreich wie eine $\mathrm{Ab}$ lehnung der fortschreitenden Digitalisierung. Einige in der Forschung identifizierten Erfolgsfaktoren werden hingegen weiterhin von hoher Relevanz sein (vgl. Lauer 2019). Den Führungskräften, der Einbindung der Beschäftigten in die Veränderungsprozesse und der Kommunikation von Veränderungen wird bei der guten Gestaltung von Veränderungen vermutlich eine noch stärkere Schlüsselrolle zukommen als dies schon in der Vergangenheit der Fall war.

Daraus ergeben sich folgende Forschungsfragen:

- Was sind neue oder verstärkte Auslöser für Wandel im Unternehmen (intern und extern)?

- Welche Veränderungen sollten von wem angestoßen werden?

- Wie sehen gute betriebliche Strukturen für Veränderungen aus?

- Wie sehen eine gute Arbeitsgestaltung und erfolgreiche Prävention im permanenten Wandel aus? 
- Welche Voraussetzungen müssen bei Unternehmen wie Beschäftigten erfüllt sein, um dem permanenten Wandel gewachsen zu sein?

\section{Agiles Arbeiten als neue Form der flexiblen Teamarbeit}

$\mathrm{Zu}$ den neuen Arbeitsformen gehören unter anderem agile Arbeitsstrukturen. Agiles Arbeiten bedeutet schnelles und selbstständiges Arbeiten im Team. Es bietet Unternehmen und Beschäftigten die Möglichkeit, schnell auf Änderungen, z. B. auf neue Kundenanforderungen, reagieren zu können. Selbstorganisierte Teamarbeit und eine gemeinsame Vision bilden die Grundlage verbunden mit schrittweiser Produktentwicklung, Transparenz, Nutzenoptimierung, agiler Führung und Feedback. Es handelt sich dabei aber nicht um beliebig flexible Formen der Arbeitsorganisation, sondern um relativ hoch strukturierte Arbeitsformen mit definierten Rollen der (Team-)Mitglieder.

Diese Art des flexiblen Arbeitens wurde Ende der 90erJahre in der Software-Entwicklung entwickelt und lange vorwiegend in dieser Branche genutzt. Nun werden einzelne Arten, je nachdem wie es die Unternehmensstrukturen ermöglichen, ebenso auf andere Branchen und Tätigkeitsbereiche übertragen. Agiles Arbeiten - zumindest Teile davon - gewinnen demnach mehr Bedeutung in der Bankenund Versicherungsbranche, in der Produktion, bei Unternehmensberatungen, in Personal- und Marketingabteilungen sowie in Schulen und im Ehrenamt. Stichworte, die sich heute in diversen Arbeitskontexten wiederfinden sind Scrum (Master), Kanban und Design Thinking. Allerdings gehen die Meinungen auseinander, ob agiles Arbeiten eher eine Denkweise (Grundhaltung/Unternehmenskultur) oder eine Methode ist bzw. ob es ausreicht, einige Methoden anzuwenden, um agil zu sein, oder nur agil gearbeitet wird, wenn das ganze Unternehmen dahingehend umstrukturiert wird.

Studien wie „Status Quo (Scaled) Agile“ aus den Jahren 2005 und 2020 zeigen (Komus 2020), dass die meisten Unternehmen eher einige Methoden des agilen Arbeitens nutzen und weniger vollständige Strukturen implementieren. Zudem werden die Erfolgsquoten agiler Ansätze von Beschäftigten deutlich positiver bewertet als die des klassischen Projektmanagements. Allerdings nähern sich die Erfolgsquoten agiler und klassischer Methoden über die Zeit hinweg an. Weitere systematische Belege zum Nutzen in den unterschiedlichsten Wirtschafts- und Dienstleistungsbereichen stehen aber bislang noch aus. Erste Forschungsarbeiten zeigen zudem, dass die Merkmale agilen Arbeitens (z.B. Autonomie in Teams und Feedback) positive Effekte auf Gesundheit und Leistungsfähigkeit von Beschäftigten haben, so sie denn gut gestaltet sind (Mitdenken 4.0 2020).
Je nach Einführung und Ausgestaltung könnte agiles Arbeiten auch Risiken bergen z.B. durch hohen Zeitdruck, hohe Arbeitsintensität, Konflikten in der Zusammenarbeit.

In Summe besteht weiterhin Unklarheit bezüglich der Gestaltungskriterien agiler Arbeit, damit diese eine gesundheitserhaltende und motivationsförderliche Wirkung entfalten können. Aktuelle Studien stammen zumeist aus der Softwareentwicklung und befassen sich nicht mit potenziellen Effekten auf Leistung und Motivation. Zudem haben diese Studien vor allem agile Teams und weniger größere, agile Strukturen wie agile Projekt- oder Prozessorganisation im Fokus.

Daraus ergeben sich folgende Forschungsfragen:

- Welche Vorteile hat agiles Arbeiten im Vergleich zu klassischer Projektarbeit in Bezug auf Kennzahlen wie Umsatz, Kundenzufriedenheit, Produktivität etc.?

- Welche Auswirkung hat agiles Arbeiten auf Gesundheit, Motivation und Leistungsfähigkeit?

- Welche Formen von agilem Arbeiten (und welcher Grad der Implementierung) sind für welche Art der Unternehmen sinnvoll? Ist ein Nebeneinander von klassischer Arbeitsorganisation und agilem Arbeiten (auch innerhalb einer Abteilung/eines Teams oder Bereichs) denkbar? Und gegebenenfalls unter welchen Voraussetzungen?

- Was gilt es bei der Einführung agiler Methoden und Mindsets auf betrieblicher Ebene zu beachten, und welche Konsequenzen ergeben sich daraus für das Management, die Führungskräfte und Mitarbeitende?

- Wie kann agiles Arbeiten in Unternehmen verschiedener Größe (von KKU bis Konzern) umgesetzt werden? Worauf ist hierbei zu achten?

- Welcher Anpassungsbedarf ergibt sich bei tariflichen Entgeltstrukturen in Bezug auf agiles Arbeiten?

\section{Die Arbeitszeit der Zukunft braucht mehr Spielräume}

Wunsch und Notwendigkeit zeitlich (noch) flexibler zu werden, werden weiterhin wachsen und mehr Bereiche durchdringen als bislang. Für Unternehmen bedeutet zeitliche Flexibilität die Anpassung an eine schwankende Auftragslage, Kundenwünsche und Unternehmenssituation. Zeitliche Flexibilität hat daher eine enorme wirtschaftliche Bedeutung. Für Beschäftigte bedeutet die Flexibilisierung eine bessere Vereinbarkeit der Lebensbereiche und mehr Zeitbzw. Handlungsspielräume. Herausforderung für Unternehmen ist es, diese „doppelte Flexibilität“, also die Flexibilisierungsanforderungen von Seiten der Unternehmen mit den Flexibilisierungsangeboten für die Beschäftigten, in Einklang zu bringen. Flexible Arbeitszeit muss so gestaltet werden, dass sie einen wirtschaftlich sinnvollen, bedarfsorientierten Personaleinsatz sicherstellt und idealerweise eben- 
so die individuellen Bedürfnisse der Beschäftigten berücksichtigt.

Nicht alle Tätigkeiten lassen vollumfängliches flexibles Arbeiten zu oder Beschäftigte verzichten bewusst auf Flexibilisierungsmöglichkeiten, um Grenzen zwischen den Lebensbereichen zu wahren. Dennoch ist es wichtig, heute schon Konzepte für die Gestaltung guter Arbeitszeit von morgen zu entwickeln, denn bislang sind diese noch nicht immer so aufgebaut, dass sie von Unternehmen und Beschäftigten gleichermaßen akzeptiert werden. Natürlich gibt es bereits verschiedene Möglichkeiten Arbeitszeit flexibler zu gestalten, z.B. durch Gleitzeit, Vertrauensarbeitszeit, Teilzeit, (Lebens-) Arbeitszeitkonten, Sabbaticals usw. Die Möglichkeiten für mehr zeitliche Flexibilisierung sind jedoch nicht ausgeschöpft. Damit Unternehmen sinnvolle und zeitgemäße Angebote unterbreiten können und gleichzeitig in der Lage sind, auf sich wandelnde Märkte zu reagieren, braucht es bessere (rechtliche) Rahmenbedingungen. Eine wöchentliche statt tägliche Höchstarbeitszeit würde es erlauben, die Verteilung der Arbeitszeit entsprechend flexibel $\mathrm{zu}$ gestalten und auf mehr oder weniger arbeitsintensive Arbeitstage besser zu reagieren, in dem die täglichen Arbeitszeiten unter der Woche variiert werden können. Arbeitgeber könnten so ihren Beschäftigten einräumen, unterschiedlich lang oder in zeitlich getrennten Blöcken zu arbeiten. In diesem Zusammenhang wäre es für die Beschäftigten und Unternehmen ein großer Gewinn an Flexibilität, wenn Ruhezeiten in verschiedene Blöcke aufgeteilt werden könnten. Diese Fragestellung lässt sich beispielsweise in Experimentierräumen klären, die aufgrund der Forschungsfrage über einen gewissen Zeitraum unabhängig vom aktuellen Arbeitszeitgesetz agieren dürfen.

Neben neuen Rahmenbedingungen für flexibleres Arbeiten nimmt auch die wirtschaftliche Bedeutung der Schichtund Nachtarbeit weiter zu. Dieser Trend wird sich mit der steigenden Nachfrage nach Dienstleistungen und dem Einsatz kapitalintensiver Produktionseinrichtungen weiter fortsetzen. Für die ergonomische, in jeder Lebensphase gesunderhaltende Schichtplangestaltung ist die Berücksichtigung arbeitswissenschaftlicher Erkenntnisse nach wie vor unerlässlich. Insbesondere vor dem Hintergrund der Digitalisierung und des demografischen Wandels erscheint es angebracht, die neu gewonnenen Ansätze und Erkenntnisse offen zu diskutieren und gegebenenfalls in die aktuellen und zukünftigen Diskussionen zu integrieren. Flexible, gesundheits- und bedarfsgerechte Schichtarbeit ist planbar, wie erste Studien verdeutlichen (vgl. Stowasser et al. 2019).

Daraus ergeben sich folgende Forschungsfragen:

- Wie sehen gute Rahmenbedingungen für eine „doppelte“ Flexibilität aus? Wie kann es Unternehmen gelingen sie umzusetzen und welche äußeren Bedingungen (z. B. auf rechtlicher Ebene) braucht es dafür?

- Wie ist eine ganzheitliche, bedarfsgerechte Arbeitszeitgestaltung bis zur Rente zu realisieren?

- Wie können die Unternehmen in die Lage versetzt werden, ganzheitliche und bedarfsgerechte Arbeitszeitmodelle zu entwickeln und ihre Arbeitsorganisation nachhaltig im Hinblick auf die zunehmend digitalisierte und dynamisierte Arbeitswelt anzupassen?

- Wie kann wöchentliche Höchstarbeitszeit und Arbeit in verschiedenen Blöcken bedarfs- und gesundheitsgerecht gestaltet werden? Wie wirkt sich diese Arbeitsgestaltung auf Leistungsfähigkeit, Motivation und Gesundheit aus?

- Wie lassen sich die arbeitswissenschaftlichen Kriterien zur Arbeitszeit- und Schichtplangestaltung mit den veränderten Anforderungen der Arbeitswelt 4.0 (besser) in Einklang bringen? Wie müssten diese arbeitswissenschaftlichen Kriterien angepasst werden?

- Inwiefern verfügen die unterschiedlichen Branchen über ausreichend Flexibilität innerhalb bestehender tariflicher Regelungen um den neuen Arbeitszeitmodellen gerecht werden zu können?

\section{Mobile Arbeit bietet noch ungenutztes Potenzial}

Neben dem zeitflexiblen gewinnt das ortsflexible Arbeiten an Bedeutung. Es erlaubt eine bessere Vereinbarkeit der Lebensbereiche und reduziert Wegezeiten. Dabei sind die Potenziale noch lange nicht ausgeschöpft. In Zukunft wird sich der Anteil der Beschäftigten, die ortsflexibel arbeiten können, noch weiter erhöhen. Der technologische Fortschritt (steigende Leistungsfähigkeit der Breitbandtechnologie, Weiterentwicklung mobiler Anwendungen und Endgeräte) ermöglicht beispielsweise ortsunabhängiges Arbeiten in der Produktion. Vor allem in Großbetrieben der Automobilindustrie zeichnet sich bereits heute ein Trend hin zu mobiler Arbeit in der Produktion ab (Büro für Technologiefolgen-Abschätzung des Deutschen Bundestages, TAB 2017). Zudem gibt es Berufsgruppen, die bereits seit längerem mobil arbeiten (Außendienstmitarbeiter, Pflegekräfte, Monteure und TechnikerInnen etc.). Digitale Kommunikationsmöglichkeiten machen heute ortsunabhängiges Arbeiten für verschiedenste Branchen möglich.

In Bezug auf ortsflexibles Arbeiten muss man folgende Unterscheidungen treffen: Telearbeit, mobile Arbeit und Homeoffice. Beim mobilen Arbeiten soll und kann die Arbeitsleistung generell von wechselnden Orten erbracht werden, wie auf einer Dienstreise, im Coworking Space oder von daheim. Homeoffice ist demnach eine Art des mobilen Arbeitens. Telearbeit hingegen ist, wenn der Arbeitgeber selbst den Arbeitsplatz in den Räumlichkeiten des Ar- 
beitnehmers fest einrichtet. Durch die Corona-Pandemie erlebt speziell Homeoffice ein gestiegenes Interesse. Im April 2020 arbeiteten laut einer Bitkom-Studie (2020) nur rund ein Drittel der Beschäftigten ganz oder teilweise im Homeoffice. Zur Bewältigung der Corona-Krise und auch im Rahmen des Infektionsschutzes stieg diese Zahl stark an. Drei Viertel der Firmen in Deutschland bieten während der Corona-Krise Homeoffice an (vgl. Litsche et al. 2020).

Barrieren bzgl. mobilem Arbeiten (oder zur Telearbeit) wie eine ausgeprägte Anwesenheitskultur oder suboptimale technische Infrastrukturen in den Unternehmen könnten durch die Corona-Krise also aufgeweicht worden sein. Der Resilienz-Check 2020 von BDA und Microsoft (2020) zeigt, dass Unternehmen flexibler geworden sind und ihre digitale Infrastruktur erweitert haben. Natürlich stellen sich in diesem Rahmen nun neue Fragen, wie z. B. Bürokonzepte aussehen, die einer steigenden Zahl an Tagen mobiler Arbeit pro Woche Rechnung tragen. Ebenso in welchem Rahmen mobile Arbeit noch vertretbar ist im Hinblick auf die gegenseitige Unterstützung durch Kollegen und Führungskräfte. In einigen Tarifverträgen haben Tarifvertragsparteien dazu bereits Regelungen implementiert und schaffen damit schon gute Rahmenbedingungen für mobile Arbeit.

Die entscheidende Kenngröße für die Bestimmung des Potenzials für mobile Arbeit ist die Möglichkeit, Tätigkeiten an unterschiedlichen Orten zu erledigen. Dabei kommt der Analyse von Tätigkeiten, Qualifikationen sowie betrieblichen, organisatorischen und persönlichen Aspekten eine wichtige Bedeutung zu. So kann festgestellt werden, für welche Tätigkeiten oder Bereiche ortsflexible Arbeit in Frage kommt und welche Qualifikationen der Beschäftigten erforderlich sind.

Es ergeben sich daher folgende Forschungsfragen:

- Welche Faktoren sind wesentlich, um das Potenzial mobiler Arbeit ebenfalls in ,krisenfreien“ Zeiten besser zu nutzen?

- Welche Aspekte sind zu betrachten, um die jeweiligen Unternehmensanforderungen mit den Wünschen der Beschäftigten abzugleichen und optimal aufeinander abzustimmen?

- Wie sehen praxistaugliche Gestaltungsmöglichkeiten zur Entwicklung und Umsetzung ganzheitlicher, mobiler Arbeit aus - insbesondere im Hinblick auf die zeitliche und inhaltliche Organisation von mobiler- und ortsgebundener Arbeit im Unternehmen?

- Wie können passende Qualifizierungskonzepte für den Umgang mit mobilem Arbeiten für Unternehmen, Führungskräfte und Mitarbeitende gestaltet werden (bzgl. neuer digitaler Technologien, virtueller Zusammenarbeit, Kooperation und Führung, der Einhaltung des Arbeitsund Gesundheitsschutzes sowie Datenschutzrichtlinien)?
- Welche Auswirkungen haben mobiles Arbeiten, Homeoffice und Telearbeit auf die Notwendigkeit von Büroflächen und auf die Gestaltung von Büroräumen?

- Wie sehen (Arbeits-)Gestaltungskompetenzen von Führungskräften und Beschäftigten aus? Welche werden benötigt?

- Wie kann eine Führungs- und Unternehmenskultur geschaffen werden, um mobile Arbeit erfolgreich umsetzen zu können?

- Welche (neuen) Belastungen ergeben sich aus orts- und zeitflexibler Arbeit (auch psychosozial)?

- Wie sehen Arbeitsstrukturen (auch orts- und zeitflexibler Zusammenarbeit) zukünftig aus?

- Wie muss Unternehmensführung und -kultur aussehen in Bezug auf Arbeiten, die mobil, ausschließlich ortsfest oder auch ,gemischt“" ausgeführt werden?

\section{Anforderungen an Führungskräfte verändern sich}

Im Zeitalter von Digitalisierung, agilem Arbeiten, neuen Arbeitsprozessen und verschiedenster Transformationen stehen Führungskräfte vor zahlreichen neuen Herausforderungen. Der permanente Wandel führt dabei auch $\mathrm{zu}$ veränderten Ansprüchen der Mitarbeitenden wie z.B. zu einem kollaborativeren, teamintegrierenden und partizipativen Vorgehen (Weinert und Ohliger 2019). Ebenso hat die Corona-Pandemie die Arbeitswelt der Führungskräfte maßgeblich verändert. Seit Beginn der Pandemie arbeiten viele Menschen nicht mehr vor Ort im Büro, was beispielsweise Führung auf Distanz stärker erforderlich macht.

In Bezug auf die neuen Anforderungen an Führungskräfte flankiert mit entsprechender Personal- und Organisationsentwicklung (vgl. hierzu auch Hinweis in Kapitel 2) ist zu klären, welche Führungsstile zukünftig verstärkt benötigt werden, welche Kompetenzen Führungskräfte benötigen, und mit welchem Mindset den Herausforderungen erfolgreich begegnet werden kann. Fest steht, dass die Bindung der Mitarbeitenden an das Unternehmen eine Grundvoraussetzung darstellt. Vertrauen, um Verantwortung abgeben zu können, Kommunikation und Motivation sind Aspekte, denen dabei eine wichtige Rolle zukommt. Die Mitarbeitenden und die Unternehmensführung müssen sich eines gegenseitigen Vertrauens sicher sein, damit die Mitarbeitenden ihre Potenziale hinreichend einbringen und nutzen können. Eine wertschätzende, mitarbeiterorientierte Führung führt zu höherer Arbeitszufriedenheit und trägt zum wirtschaftlichen Erfolg bei. Daraus folgt, dass die Strategie eines Unternehmens mit dem Personalmanagement zukünftig immer stärker verzahnt sein wird. Personalentwicklung und Kommunikation werden so zur zentralen Führungsaufgabe (Enste et al. 2020). 
Für Führungskräfte bedeutet die neue Arbeitswelt grundsätzlich ein Austarieren zwischen der Einführung neuer Arbeitsformen (z. B. agilen Methoden) und dem Weiterbestehen von klassischen Organisationselementen (z.B. regelmäßige Teammeetings oder Feedbackgespräche). Das neue Führungsverständnis erfordert, eine Umgebung zu schaffen, in der Teams ihre Zusammenarbeit und Prozesse kontinuierlich verbessern können. Beim mobilen Arbeiten ist es beispielsweise wichtig, auf klare Strukturen und noch engeren Kontakt zu den Mitarbeitenden zu setzen. Dies bedingt neben neuen Kommunikationswegen auch einen höheren Organisationsaufwand. Die Führungskraft sollte die Arbeitsabläufe so organisieren, dass sie dem ganzen Team zur Erreichung der Ziele dienen und beidseitige Erwartungshaltungen von Führungskraft und Mitarbeitenden in Einklang gebracht werden.

Weiterhin spielt das Thema „Nachhaltigkeit“ im Bereich Führung eine wichtige Rolle. Dabei umfasst Nachhaltigkeit mehr als ökologische Aspekte. Nachhaltige Führung bedeutet neben fachlichen Ergebnissen und der Zufriedenheit der Mitarbeitenden vor allem effizienten Einsatz von Ressourcen und den Erhalt langfristiger Leistungsfähigkeit. Ziel ist es, die Arbeitsfähigkeit (das Können) und die Motivation (das Wollen) der Mitarbeitenden zu erhalten bzw. zu fördern und dadurch die Innovations- und Zukunftsfähigkeit eines Unternehmens zu erhöhen. Dieser Wandel hin zu einer nachhaltigen Struktur ist jedoch ein langandauernder Prozess.

Daraus ergeben sich folgende Forschungsfragen:

- Welche Führungsstile werden zukünftig für welche Aufgaben/Situationen verstärkt benötigt?

- Welche Kompetenzen benötigen die Führungskräfte und mit welchem Mindset von Führungskräften und Mitarbeitenden kann den Herausforderungen erfolgreich begegnet werden?

- Was macht eine nachhaltige und ganzheitliche Führung aus?

\section{Eigenverantwortung wird immer wichtiger}

Arbeit kann heute eigenverantwortlicher bzw. selbstbestimmter gestaltet werden und kann Gesundheit und Wohlbefinden fördern. Ein höherer Grad an Autonomie kann zu weniger Erschöpfung und besserem Abschalten von der Arbeit führen. Viele Unternehmen verwenden seit einigen Jahren ergebnisorientierte Führungskonzepte, welche darauf basieren, Ziele zu vereinbaren, und den Erfolg entsprechend zu honorieren, statt Aufgaben zu vergeben, Der Weg zur Zielerfüllung ist den Beschäftigten dabei in einem bestimmten Rahmen frei; sie können eigenverantwortlich und in gewissen Maßen selbstständig die vereinbarten
Ziele erreichen. Bei dieser Form der Steuerung steht demnach eigenverantwortliches, selbstbestimmtes Handeln der Beschäftigten im Mittelpunkt.

Zusammen mit der Selbstbestimmung wächst die Rolle der Arbeitsgestaltungskompetenz. Darunter wird die Kompetenz verstanden, die eigene Arbeit so zu gestalten, dass man selbst gesund, motiviert und leistungsfähig bleibt. Der Arbeitgeber muss natürlich zu arbeitsschutzrelevanten Themen unterweisen und kann zusätzlich Workshops anbieten bzw. Arbeitsmittel wie einen Laptop zur Verfügung stellen. Auf eine adäquate und ergonomische Sitzposition, Blendungsfreiheit etc. muss der Beschäftigte selbst an seinem gewählten Arbeitsort achten. Aus der Möglichkeit, als Beschäftigter die eigene Arbeit aktiv zu gestalten, ergibt sich auch die Notwendigkeit dies zu tun. Mehr Selbstbestimmung und Eigenverantwortung können daher zu einer Herausforderung und einem Belastungsfaktor werden, denn mehr Autonomie bei der Arbeit erfordert zusätzliches Selbstmanagement und Koordination.

Damit Beschäftigte eigenverantwortlich agieren können, müssen daher die Rahmenbedingungen im Unternehmen stimmen. Elementar sind dabei (1) Strukturen und Kultur, (2) Führung und (3) Qualifizierung und Befähigung. Bestimmte Strukturen, wie gelebte Verbesserungsprozesse und eine transparente Fehlerkultur, begünstigen die Übernahme von Eigenverantwortung. Die Kultur eines Unternehmens sollte das eigenverantwortliche Handeln der Beschäftigten fördern. Dies beinhaltet ebenfalls alternative und unkonventionelle Lösungen (jedoch nicht in Bezug auf sicherheitsrelevantes Verhalten). Das Verhalten der Führungskräfte spielt ebenso eine zentrale Rolle, vor allem regelmäßiges und konstruktives Feedback. Führungskräfte müssen zudem Verantwortung abgeben können, damit ihre Mitarbeiter sich entsprechend einbringen können. Weitere Voraussetzungen für das „Sich-Einbringen-Können“ sind Qualifizierung und Befähigung der Beschäftigten u. a. zum eigenständigen Lernen sowie zum Erlangen beruflicher Handlungskompetenz. Das Wissen und Können der Beschäftigten kann von Seiten des Arbeitgebers durch geeignete Maßnahmen geschult werden, z.B. durch Unterweisungen, welche auf der Gefährdungsbeurteilung fußen.

Daraus ergeben sich folgende Forschungsfragen:

- Wie können Arbeitsgestaltungskompetenz und Eigenverantwortung im Unternehmen gefördert und arbeitgeberseitig eingefordert werden?

- Welche Rahmenbedingungen von Seiten der Unternehmen braucht es, um die Arbeitsgestaltungskompetenz passend zu flankieren? Wie können Führungskräfte entsprechend befähigt werden?

- Wie können passende Trainingskonzepte zur Entwicklung von Arbeitsgestaltungskompetenz und Eigenverantwortung gestaltet werden? 
- Welche Art der Unterstützung brauchen Kleinst- und Kleinunternehmen bei der Umsetzung bestimmter Themen, z.B. der Gefährdungsbeurteilung auch zur psychischen Belastung?

\section{Nachhaltigkeit als zentrales Element des Wirtschaftens}

Die Enquete-Kommission „Schutz des Menschen und der Umwelt" des Bundestages entwickelte 1995 das DreiSäulen-Modell einer nachhaltigen Entwicklung, welches den Begriff Nachhaltigkeit interdisziplinär beschreibt: Die Säulen „Ökologie“, „Soziales“ und „Wirtschaft" stehen in Wechselwirkung zueinander und müssen für eine dauerhaft zukunftsfähige Entwicklung gemeinschaftlich betrachtet werden. Obwohl das Säulen-Modell immer wieder kritisiert wird, hat sich bisher kein anderes Modell durchsetzen können. Auch kommt kaum ein Modell ohne den Dreiklang Ökologie-Soziales-Wirtschaft aus, sodass über diesen Dreiklang als Ausgangspunkt für die Nachhaltigkeitsdiskussion und die langfristige Betrachtung einer nachhaltigen Entwicklung ein Konsens angenommen werden kann (Deutscher Bundestag 13. Wahlperiode 1998).

Auf gesellschaftlicher Ebene beschreibt Nachhaltigkeit eine Politik, deren Ziel es ist, ökologische, ökonomische und soziale Leistungsfähigkeit - unterstützt durch eine moderne Technologie - sicherzustellen und sogar zu verbessern. Auf betrieblicher Ebene heißt Nachhaltigkeit, die Unternehmenssituation bereits heute zu verbessern, ohne dadurch die langfristigen Zukunftsperspektiven zu verschlechtern. In den modernen Managementsystemen verschmelzen die Anforderungen aus gesellschaftlicher und betrieblicher Ebene zu den Anforderungen der sogenannten Anspruchsgruppen (engl.: Stakeholder). Daher lässt sich in modernen Managementsystemen ebenso die Auseinandersetzung mit dem Nachhaltigkeitsbegriff finden. Auch aus diesem Grund haben sich bereits viele Unternehmen den Gedanken der Nachhaltigkeit zugewandt.

Das Einbeziehen von Nachhaltigkeitsstrategien und das Abwägen geeigneter Maßnahmen, wie z.B. die Implementierung abfallarmer und klimafreundlicher Arbeitsschritte in der Produktion oder die Einführung von „Nachhaltigkeits-Guidelines" bei Einkauf und Beschaffung sowie der Produktentwicklung, ergänzen und erweitern bestehende Unternehmensprozesse. Diese Veränderungen betreffen jede Ebene in Unternehmen und sind somit für Beschäftige und Führungskräfte relevant. Ferner resultiert aus diesen Veränderungen ein Bedeutungszuwachs an vernetzter, interdisziplinarer und holistischer Zusammenarbeit, um der Komplexität des Nachhaltigkeitsbegriffs gerecht zu werden. Aller Wahrscheinlichkeit nach werden sich daher die Anforderungsprofile der Tätigkeiten in Bezug auf den
Aspekt Nachhaltigkeit verändern. Der Bedarf an veränderten oder neuen Berufsbildern, Fachkräften mit spezieller Ausbildung oder zugeschnittenen Fort- und Weiterbildungsformaten wird steigen (Ellen MacArthur Foundation 2015).

In den letzten Jahren verstärkt die Politik den Druck auf Unternehmen, nicht nur die innerbetrieblichen Prozesse anzupassen, sondern auch ihre, nach außen wirkende, Verantwortung wahrzunehmen (Europäische Kommission 2019, 2020). Dabei stehen zusätzlich zur Versorgungssicherheit weitere Aspekte wie der Umwelt- und Klimaschutz, Fairness und gute Arbeitsbedingungen sowie die Auswirkungen von Digitalisierung und KI auf eine zukunftsfähige Arbeitsgestaltung im Vordergrund. Flankiert wird diese Entwicklung durch den zunehmenden wissenschaftlichen Erkenntnisgewinn, nachhaltiger wirtschaften zu müssen sowie den gesellschaftlichen Wertewandel.

Daraus ergeben sich folgende Forschungsfragen:

- Wie werden in Zukunft Nachhaltigkeitsstandards und -anforderungen definiert? Welche Auswirkung haben die Komplexität des Themas Nachhaltigkeit und wissenschaftlicher Fortschritt auf Unternehmen unterschiedlicher Größen und deren Beschäftigte? Wie wird hierdurch auch der Mensch in seinen verschiedenen Rollen (Verbraucher, Arbeitnehmer, Führungskraft) beeinflusst werden?

- Wie verändern neue Treiber wie z.B. die Nachhaltigkeit den Arbeitsmarkt?

- Wie werden neue und bestehende Geschäftsmodelle im Einklang, Umwelt und Gesellschaft (weiter-) entwickelt? Welche Auswirkungen können neue Geschäftsmodelle auf die Arbeitsgestaltung und Arbeitsbedingungen haben?

- Wie wirken sich Nachhaltigkeitsbeschlüsse der Politik - sowohl auf nationaler als auch internationaler Ebene auf die Unternehmen und deren Beschäftigte aus? Beschleunigen diese Anforderungen den Wandel Richtung flexibler, digitaler Arbeitsgestaltung und neuer Technologien? Können traditionelle Berufsbilder und Technologien in der Nachhaltigkeitsdiskussion mithalten oder ergänzen sie diese sogar? Welche Potenziale können (müssen) durch politische Nachhaltigkeitsbeschlüsse freigesetzt werden?

- Wie sehen Arbeitsstrukturen unter Berücksichtigung der Nachhaltigkeitsdiskussion zukünftig aus?

- Wie robust sind Wertschöpfungsketten? Wie bedingen sich hier Anpassungsbedarfe unter-schiedlicher Wandlungsprozesse (Digitalisierung, KI, globale vs. lokale Ketten, etc.)? 


\section{Digitalisierung und Künstliche Intelligenz bieten Chancen für gute Arbeitsgestaltung}

Die Themen Digitalisierung und künstliche Intelligenz (KI) erleben seit einigen Jahren einen Boom in der Forschung, und verschiedene nationale und internationale Fördermittelgeber haben umfangreiche Mittel für Forschungsfragen bereitgestellt, die wie z.B. im Rahmen des Forschungsprogramms „Zukunft der Arbeit“ des Bundesforschungsministeriums auch explizit die Arbeitsforschung umfassen. Dies ist nach vielen Jahren, in denen der Arbeitsforschung keine größere Aufmerksamkeit geschenkt wurde, zu begrüßen. Der Forschungsbedarf bleibt dennoch hoch, zum einen, weil noch nicht alle wichtigen Forschungsfragen untersucht werden konnten, zum anderen, weil die Digitalisierung und die Anwendung künstlicher Intelligenz in den Unternehmen weiter voranschreiten und kontinuierlich neue Forschungsfragen aufwerfen. Die arbeitspolitische Gestaltung beeinflusst wesentlich den Erfolg von Digitalisierung und KI. Daher sind die sachliche Debatte und der Dialog über Mensch und Arbeit im Kontext der Digitalisierung und KI mit allen Beteiligten (Beschäftigten, Unternehmen, Politik und Sozialpartnern) notwendig.

Grundsätzlich ist zu berücksichtigen, dass Unternehmen und/oder ihre Kunden von den bestehenden Entwicklungen unterschiedlich stark betroffen sein werden und sich technologische Entwicklungen bei Produkten und Prozessen in unterschiedlicher Intensität und Geschwindigkeit auswirken. Forschung darf nicht nur die besonders betroffenen Unternehmen und Vorreiter in den Blick nehmen. Genauso wichtig ist es, wirtschaftliche Potenziale und Potenziale für gute Arbeitsgestaltung und eine stärkere Einbindung von leistungsgewandelten Menschen auch für Unternehmen, die unter einem weniger starken Änderungsdruck stehen, aufzuzeigen.

Die Forschung muss sich stark an den Praxisbedarfen und einer zügigen Umsetzung orientieren. Kooperation zwischen Wirtschaft und Wissenschaft ist elementar, um die Potenziale von Digitalisierung und KI zu heben. Kontrollierte Experimentierräume und arbeitswissenschaftliche Begleitprojekte erweisen sich in der betrieblichen Praxis als zweckmäßig, um a) neue Technologien im Feld zu erproben und zu evaluieren sowie b) umfassende Akzeptanz zur Verwendung von Digitalisierungs- und KI-Lösungen zu erzielen. Insofern ist es mit Sorge zu betrachten, dass gemäß einer Studie des Instituts der Deutschen Wirtschaft der ohnehin schon geringe Anteil von gut drei Prozent an wissenschaftlichen Publikationen in Deutschland zu KI, die in Zusammenarbeit von Wissenschaft und Unternehmen entstanden, sogar noch weiter abnimmt (Klös und Schäfer 2020). Dabei genügt Technologieforschung allein nicht. Arbeits- wissenschaftliche Aspekte haben eine hohe Bedeutung für die Akzeptanz und den Einsatzerfolg neuer Technologien.

Daraus ergeben sich folgende Forschungsfragen:

- Welche Rolle soll der Mensch in der veränderten Arbeitswelt einnehmen? Wie wird sich die Aufgabenteilung und ggf. Interaktion zwischen Mensch und Maschine bzw. Mensch und KI entwickeln?

- Inwieweit wird der Mensch weiterhin steuernde, durchführende und überwachende Tätigkeiten ausführen?

- Wie verändern die Digitalisierung und der Einsatz von KI typische Aufgaben und Berufe in den Unternehmen?

- Können technologische Entwicklungen dem mit dem demografischen Wandel verbundenen Fachkräftemangel entgegenwirken?

- Wie können neue Technologien Chancen für leistungsgewandelte Beschäftigte heben?

- Wie kann Arbeit weiterhin gut und gesundheitsgerecht gestaltet werden?

- Wie können die Befürchtungen der Beschäftigten gegenüber neuen Technologien genommen werden?

\section{Zentrale Thesen zur Richtung der Arbeitswelt}

1. Eine zunehmende Anzahl an Unternehmen unterliegt einem permanenten Wandel. Für diese Unternehmen greifen Erkenntnisse über klar abgegrenzte Veränderungsprozesse mit längeren Phasen ohne Veränderungen nicht mehr. Gute Arbeitsgestaltung und erfolgreiche gesundheitliche Prävention sind auch im permanenten Wandel möglich.

2. Agile Arbeit bzw. die Nutzung auch einzelner agiler Arbeitsmethoden können gesundheitserhaltend wirken, wenn entsprechende Rahmenbedingungen im Unternehmen vorhanden sind. Sie kann zudem förderlich für Motivation, Leistung und Produktivität sein. Aktuell fehlt es jedoch noch an validen Befunden und geeigneten Handlungsempfehlungen.

3. Neben klassischen Aufbauorganisationen existieren agile Organisationselemente parallel. Die Aufgabe der Führung ist es, die passenden Einsatzgebiete von agiler Führung zu analysieren, einzusetzen und dabei Konfliktpotenziale so gering wie möglich zu halten, damit sich das Unternehmen an Veränderungen schnell anpassen kann. Sie unterstützt Mitarbeiter auf Augenhöhe dabei, gemeinsam die besten Lösungen für Herausforderungen zu finden.

4. Flexible und individuelle Arbeitszeitgestaltung, welche sowohl für Unternehmen als auch für Beschäftigte vorteilhaft ist, berücksichtigt zwei Perspektiven: Zum einen muss die betriebliche Flexibilität stets zuverlässig 
Kundenbedürfnisse erfüllen. Zum anderen soll sie die zeitliche Flexibilität der Beschäftigten unterstützen und lebenssituationsspezifische Arbeitszeit ermöglichen, die z.B. die Betreuung von Kindern oder die Pflege kranker Angehöriger erlaubt.

5. Die Bedeutung der Schicht- und Nachtarbeit nimmt weiter $\mathrm{zu}$, auch aufgrund steigender Nachfrage nach Dienstleistungen und dem Einsatz kapitalintensiver Produktionseinrichtungen. Schichtplangestaltung auf der Basis arbeitswissenschaftlicher Kriterien hat einen wesentlichen Einfluss auf die Gesundheit und Leistungsfähigkeit der Beschäftigten. Gesunde und flexible Schichtmodelle sind planbar.

6. Mobiles Arbeiten gewinnt weiterhin an Bedeutung und trägt bei richtiger Gestaltung zu einer besseren Vereinbarkeit der Lebensbereiche bei und ist somit ein wesentlicher Attraktivitätsfaktor für deutsche Unternehmen im Wettbewerb um Fachkräfte. Es ist davon auszugehen, dass sich in Zukunft der Anteil der Beschäftigten, die mobil arbeiten werden, noch weiter erhöhen wird.

7. Mobiles Arbeiten bietet noch ungenutztes Potenzial für verschiedene Branchen. Die Digitalisierung, insbesondere die Datenverfügbarkeit, die Sensorik und Vernetzung von Maschinen sowie die Möglichkeiten der Fernsteuerung und Fernwartung ermöglichen, dass auch Tätigkeiten in der Produktion zunehmend flexibilisiert und somit auch ortsunabhängig ausgeführt werden können. Es gibt allerdings kein Patentrezept für Unternehmen und Beschäftigte, welches diese neue Art des Arbeitens allgemeingültig ermöglicht.

8. Die Unternehmen tragen sowohl eine nach innen als auch nach außen gerichtete Verantwortung in Bezug auf Nachhaltigkeit. Eine authentische Darlegung dieses Verantwortungsbewusstseins wird durch unterschiedlichste Einflüsse (politisch - gesellschaftlich - wissenschaftlich) weiter an Bedeutung gewinnen.

9. Anforderungen an Tätigkeiten werden sich durch das steigende Nachhaltigkeitsbewusstsein verändern. Um der Komplexität, welche mit der Nachhaltigkeitsdiskussion einher geht, gerecht zu werden, werden sich veränderte Berufsbilder, Fachkräfte mit spezieller Ausbildung und entsprechende Qualifizierungsangebote etablieren (müssen).

10. Die Bedeutung von Arbeitsgestaltungskompetenzen wächst. Eine zunehmende Autonomie oder Eigenverantwortung der Beschäftigten bei der Gestaltung von Arbeitszeit, -ort, -aufgaben und -abläufen geht mit einem „Mehr“ an Verantwortung für die eigene Gesundheit einher. Jeder Beschäftigte kann seine Leistungsfähigkeit z.B. durch konsequentes, lebenslanges Lernen und gesundheitsbewusstes Verhalten stark beeinflussen, fördern und langfristig erhalten. Entsprechende Quali- fizierungsangebote und passende Rahmenbedingungen der Unternehmen tragen dazu bei.

11. Potenziale durch KI bestehen in einer neuen Arbeitsqualität, die z.B. die Menschen mit assistierenden Systemen unterstützt, Ressourcen effektiver einsetzt, die Gesundheitsressourcen fördert oder die Prozesse umweltschonender gestaltet.

12. Durch die Digitalisierung entstehen zahlreiche Wege zur Neugestaltung von Arbeit und damit auch Potenziale für die Ergonomie und den Arbeitsschutz. Assistenzsysteme (Datenbrillen, Tablets, Smart Watches u.v.a.), technische Unterstützungsmöglichkeiten (Mensch-Roboter-Kollaborationen, Exoskelette) und weitere Automatisierungsmechanismen werden die Arbeit der Zukunft prägen.

Kernautoren (alphabetisch) Pia Barth (BDA - Bundesvereinigung der Deutschen Arbeitgeberverbände), Dr. Elisa Clauß (BDA), Julia Eckert (Gesamtverband textil+mode), Sven Hille (ifaa - Institut für angewandte Arbeitswissenschaft), Dr. Stephan Sandrock (ifaa), Prof. Dr. Sascha Stowasser (ifaa), Dr. habil. Birgit Verworn (BDA), Reinhard Walleter (Südwestmetall)

Weitere Mitwirkende Dr. Alexander Böhne (Arbeitgeberverband des privaten Bankgewerbes), Klaus Depner (Randstad Deutschland GmbH \& Co. KG), Thomas Köpp (Südwestmetall), Eckhard Metze (KAN Kommission Arbeitsschutz und Normung), Cordula Miosga (Arbeitgeberverband Region Braunschweig), Dr. Uwe Nickel (ADAC)

\section{Literatur}

Albach H, Meffert H, Pinkwart A, Reichwald R (Hrsg) (2014) Management of permanent change. Springer Gabler, Wiesbaden

BDA, Microsoft (2020) Resilienz-Check 2020. https://news.microsoft. com/wp-content/uploads/prod/sites/40/2020/09/ResilienzCheck2020.pdf. Zugegriffen: 16. Nov. 2020

Bitkom (2020) Homeoffice in Zeiten der Corona-Pandemie. www. bitkom.org/Presse/Presseinformation/Corona-Pandemie-Arbeitim-Homeoffice-nimmt-deutlich-zu. Zugegriffen: 16. Nov. 2020

Büro für Technikfolgen-Abschätzung beim Deutschen Bundestag (TAB) (2017) Chancen und Risiken mobiler und digitaler Kommunikation in der Arbeitswelt. TAB, Berlin

Deutscher Bundestag 13. Wahlperiode (1998) Abschlussbericht der Enquete-Kommission „Schutz des Menschen und der Umwelt - Ziele und Rahmenbedingungen einer nachhaltig zukunftsverträglichen Entwicklung“. Drucksache 13/11200. http://dip21. bundestag.de/dip21/btd/13/112/1311200.pdf. Zugegriffen: 17. Nov. 2020

Ellen MacArthur Foundation (2015) Growth within: a circular economy vision for a competitive Europe. www.ellenmacarthurfoundat ion.org/assets/downloads/publications/EllenMacArthurFounda tion_Growth-Within_July15.pdf. Zugegriffen: 17. Nov. 2020

Enste D, Kürten L, Suling L, Orth A (2020) IW-Analysen 135: Digitalisierung und mitarbeiterorientierte Führung. Die Bedeutung der Kontrollüberzeugung für die Personalpolitik. www.econstor. eu/bitstream/10419/214160/1/1690064986.pdf. Zugegriffen: 17. Nov. 2020

Europäische Kommission (2019) Der europäische Grüne Deal. https:// eur-lex.europa.eu/resource.html?uri=cellar:b828d165-1c22-11ea8c1f-01aa75ed71a1.0021.02/DOC_1\&format=PDF. Zugegriffen: 17. Nov. 2020 
Europäische Kommission (2020) Ein neuer Aktionsplan für die Kreislaufwirtschaft - Für ein saubereres und wettbewerbsfähigeres Europa. https://eur-lex.europa.eu/resource.html?uri=cellar: 9903b325-6388-11ea-b735-01aa75ed71a1.0016.02/DOC_1\& format=PDF. Zugegriffen: 17. Nov. 2020

ifaa - Institut für angewandte Arbeitswissenschaft, Stowasser S, Altun U, Hartmann V, Hille S, Sandrock S (Hrsg) (2019) Gutachten zur Mobilen Arbeit. Erstellt im Auftrag der Bundestagsfraktion der Freien Demokratischen Partei (FDP). ifaa, Düsseldorf

Klös HP, Schäfer H (2020) IW-Report 19/2020. IW-Arbeitsmarktmonitoring April 2020. https://www.iwkoeln.de/fileadmin/user_ upload/Studien/Report/PDF/2020/IW-Report_2020_Arbeitsmarkt monitoring_April.pdf. Zugegriffen: 17. Nov. 2020
Komus A (2020) Status quo (scaled) agile. www.hs-koblenz.de/bpmlabor/status-quo-scaled-agile-2020. Zugegriffen: 17. Nov. 2020

Lauer T (2019) Change Management - Grundlagen und Erfolgsfaktoren. Springer Gabler, Berlin, Heidelberg

Litsche S, Sauer S, Wohlrabe K (2020) Konjunkturumfragen im Fokus: Coronakrise trifft deutsche Wirtschaft mit voller Wucht. Ifo Schnelld 73(5):57-61

Mitdenken 4.0 (2020) Agiles Arbeiten. www.certo-portal.de/mitdenken 4null/agiles-arbeiten/. Zugegriffen: 17. Nov. 2020

Weinert S, Ohliger K (2019) Zwischen Wunsch und Wirklichkeit. Personalwirtschaft 12:56-58 